\title{
New Approach to Diffuse Scattering in Complex Chalcogenides
}

\author{
Dietrich Häussier ${ }^{1}$, Viola Düppel ${ }^{2}$, Marc Schlosser $^{3}$, A. Pfitzner ${ }^{3}$, and Lorenz Kienle ${ }^{1}$ \\ 1. Institute for Materials Science, Christian-Albrechts-University Kiel, Kaiserstrasse 2, 24143 Kiel, \\ Germany \\ 2. Max Planck Institute for Solid State Research Stuttgart, Heisenbergstrasse 1, 70569 Stuttgart,Germany \\ 3. Institute of Inorganic Chemistry, University of Regensburg, 93040 Regensburg, Germany
}

Earth abundant materials and low-cost fabrication are utmost criteria for future photovoltaic technologies [1]. Mixed main-group transition-metal chalcogenides fulfill these requirements, they are prospective important thermoelectric materials and useful for thin-film solar cells [2]. As an example, Cu3BiS3 thin films have shown promising structural and optical properties for special use in photovoltaics $[3,4]$. Despite exhibiting these promising material characteristics, thin film photovoltaic cells based on Cu3BiS3 have not yet reached the device level. This is mainly because the fundamental physical properties of $\mathrm{Cu} 3 \mathrm{BiS} 3$ are not yet well understood, and the quality of the films is not optimized [5]. Open-ended questions also exist along the lines of $\mathrm{Cu}_{3} \mathrm{BiSe}_{3}$. A characteristic structure-related phenomenon not yet understood are circular diffuse scattering features in electron and X-ray diffraction. They are found not only for $\mathrm{Cu}_{3} \mathrm{BiSe}_{3}$ but for instance in $\mathrm{La}_{0.70} \mathrm{Al}_{0.14} \mathrm{I}_{0.86}$ [6] and $\mathrm{K}_{2} \mathrm{In}_{12} \mathrm{Se}_{19}$ [7], too.

Diffuse scattering in X-ray and electron diffraction of multi-component systems often is discussed since several years to be related to short-range order phenomena [8,9]. But, up to now, for a lot of systems quantitative evaluations are missing.- Unlike X-ray diffraction, high-resolution TEM - essentially not combined with selected-area electron diffraction - benefits uncovering of interrelations between diffuse scattering phenomena and nanostructure. We found diffuse rings around fundamental reflections in $\mathrm{X}$-ray and (precession) electron diffraction (PED) of $\mathrm{Cu}_{3} \mathrm{BiSe} 3$.During the further procedure, highresolution TEM micrographs taken at a Philips CM30 ST $(300 \mathrm{kV})$ have been Fourier filtered. As a result, for several zone axes a relationship between those diffuse rings and circular, irregularly arranged, $2 \ldots \quad 3 \mathrm{~nm}$ sized disordered regions is proved (Figs. 1, 2). Contrary to the original, they discern clearly from the background. For certain orientations dislocation cores are visualized in the FFTs in close neighborhood of the disordered regions. Easy geometric models exhibit when Fourier transformed very similar diffuse rings around reflections this way establishing the assumption (Fig. 3).

\section{References:}

[1] F Mesa et al., Appl. Phys. Lett. 96, (2010) p. 082113.

[2] L Kienle et al., Eur. J. Inorg. Chem. 2010, p. 367.

[3] F Mesa, A Dussan, and G Gordillo, Phys. Status Solidi C 7, (2010) p. 917.

[4] V Estrella, M T S Nair, and P K Narr, Sermcond. Sci. Technol. 18, (2003) p. 190.

[5] M Kumar and C Persson, Applied Physics Letters 102, (2013) p. 062109.

[6] T Weber et al., Acta Cryst. A64, (2008) p. 641.

[7] L Kienle, A Simon, J Solid State Chem. 161, (2001) p. 385.

[8]T R Welberry in „Local structure from diffraction“ ed. S J L Billinge and M F Thorpe (1998, Plenum Press, New York and London) p. 35.

[9] K Takezawa, H Hoshi, and K Marukawa, Int. J. Materials and Product Technology, Special Issue, SPMI, (2001) p. 38. 

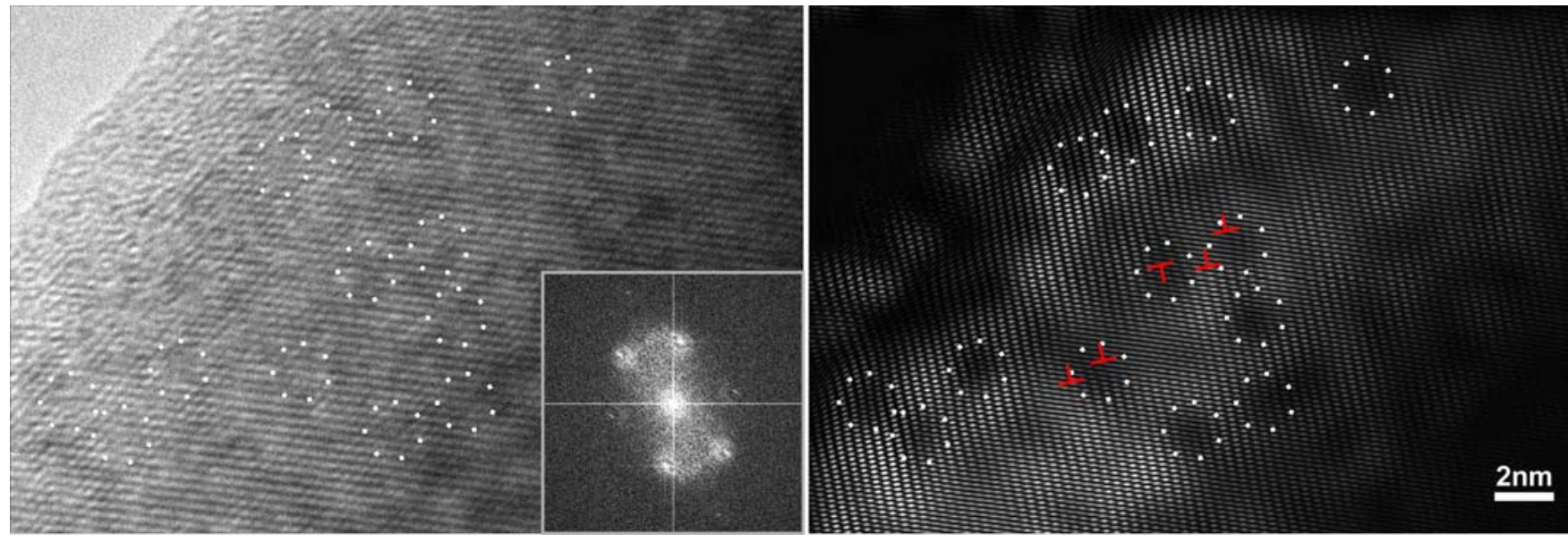

Figure 1. HR-TEM image, FFT (inset), and adaptively filtered inverse FFT. Part of disordered regions and dislocations is marked.
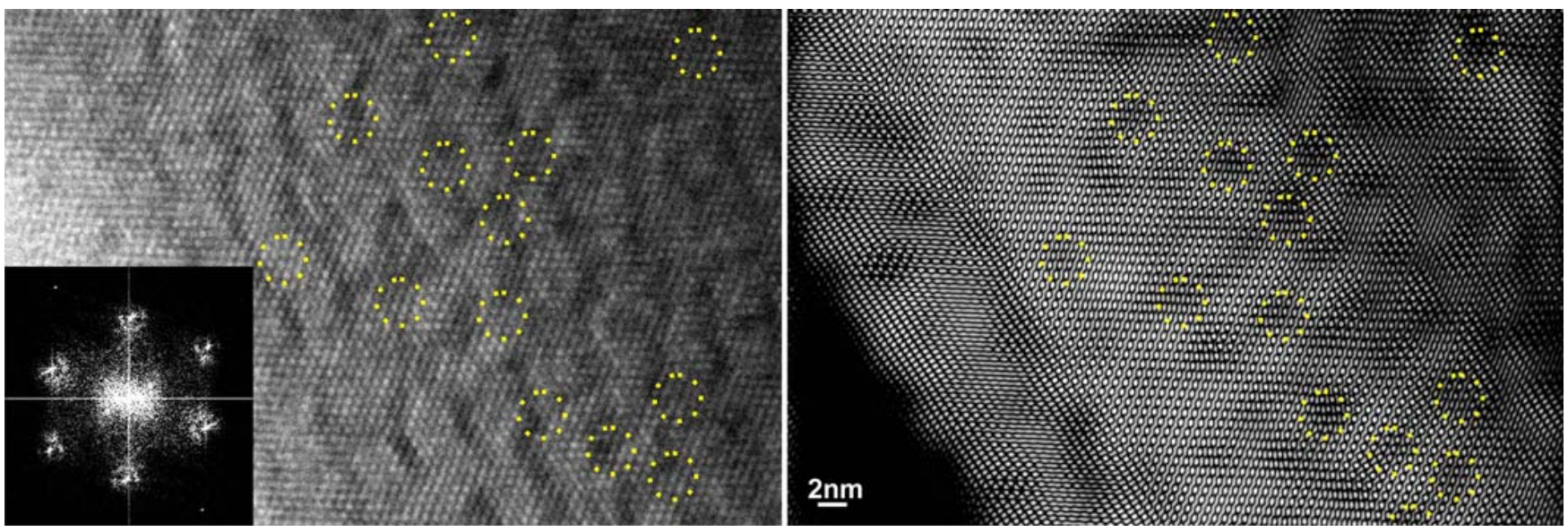

Figure 2. HR-TEM image, FFT (inset), and adaptively filtered inverse FFT. On various occasions disordered regions are marked.

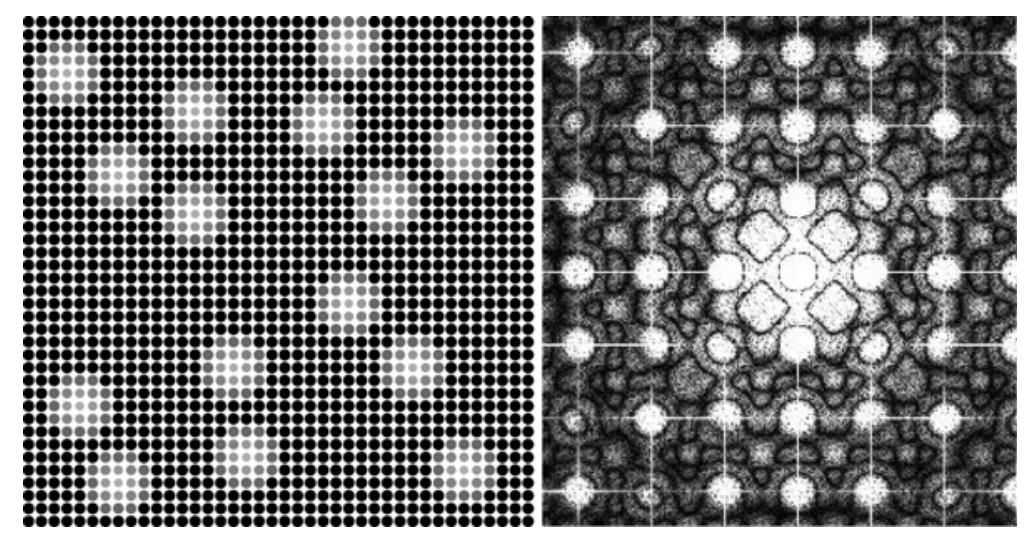

Figure 3. 2D model pattern with modified circular regions, FFT (diffuse rings). 